\title{
Information: Problems, Paradoxes, and Solutions
}

\author{
Mark Burgin
}

Department of Mathematics, University of California, Los Angeles, 405 Hilgard Ave., Los Angeles, CA 90095, USA; mburgin@math.ucla.edu

\begin{abstract}
The information age is upon us and the main paradox is that there is no satisfactory and commonly accepted answer to the crucial question what information is. This results in a quantity of contradictions, misconceptions, and paradoxes related to the world of information. We consider the existing situation in information studies, which is very paradoxical and inconsistent, in the first part of this paper. To remedy the situation, a new approach in information theory, which is called the general theory of information, is developed. The main achievement of the general theory of information is explication of a relevant and
\end{abstract}

\begin{abstract}
adequate definition of information. This theory is built on an axiomatic base as a system of two classes of principles and their consequences. The first class consists of the ontological principles, which are revealing general properties and regularities of information and its functioning. Principles from the second class explain how to measure information.
\end{abstract}

Keywords: information, general theory of information

\section{Introduction}

As Goguen writes (1997), "we live in an "Age of Information," but it is an open scandal that there is no theory, nor even definition, of information that is both broad and precise enough to make such an assertion meaningful." We are overwhelmed with myriad of information from a wide spectrum of information sources, such as the World Wide Web, emails, images, speeches, documents, etc. At the same time, our experience demonstrates that common sense understanding of the notion of information may be very misleading. Consequently, we have to go to information science and develop theoretical perspective on entertainment. The main problem is to find the right theory.

Looking into information science, we encounter a peculiar situation. On one hand, it has a lot of theories, a diversity of results, and even a proclaimed success. Scientists created a diversity of information theories: statistical or Shannon's, semantic, algorithmic, qualitative, dynamic and so on. On the other hand, as it is written in the introduction to the authoritative book "Information Policy and Scientific Research" (1986), "Our main problem is that we do not really know what information is."

To find a relevant answer, it is necessary to have an adequate and comprehensive theory of informa tion. However, many express an opinion that it is impossible to build such a theory and definition. Thus, Agre (1995) argues that the notion of information is itself a myth, mobilized to support certain institutions, such as libraries. Bowker (1994) discusses other mythologies that support the notion of information. Capurro (Capurro/Fleissner/Hofkirchner 1999) gives an informal proof of the, so-called, Capurro trilemma that implies impossibility of a unified theory of information. According to his understanding, information may mean the same at all levels (univocity), or something similar (analogy), or something different (equivocity). In the first case, we lose all qualitative differences, as for instance, when we say that e-mail and cell reproduction are the same kind of information process. Not only the "stuff" and the structure but also the processes in cells and computer devices are rather different from each other. If we say the concept of information is being used analogically, then we have to state what the "original" meaning is. If it is the concept of 
information at the human level, then we are confronted with anthropomorphisms if we use it at a non-hut man level. We would say that "in some way" atoms "talk" to each other, etc. Finally, there is equivocity, which means that information cannot be a unifying concept any more, i.e., it cannot be the basis for the new paradigm. Problems of a unified theory of information are also discussed in other publications of Capurro, Fleissner, Fuchs, and Hofkirchner.

However, the advancement of science is very fast and a new theory recently appeared. It is called the general theory of information (Burgin, 1997; 1997a). It comprises all other known theories of information and contains much more.

There have been a lot of discussions and different approaches have been suggested trying to answer the question what information is. According to Flückiger (1995), in modern information theory a distinction is made between structural-attributive and functional-cybernetic types of theories. While representatives of the former approach conceive information as structure, like knowledge or data, variety, order, and so on; members of the latter understand information as functionality, functional meaning or as a property of ofganized systems.

The principal achievement of the general theory of information is that it explains and determines what information is. The new approach changes drastically our understanding of information, i.e. of one of the most important phenomena of our world. It displays that what people call information is, as a rule, only a container of information but not information itself. This theory reveals intrinsic relations between matter, knowledge, energy, and information.

An interesting feature of this theory is that it does not contradict the Capurro trilemma, but solves this paradox. It is achieved through asserting that information is relative and introducing a systemic (not numerical) parameter that allows one to have the whole diversity of information manifestations comprised in a unified definition. This systemic parameter is a specific infological subsystem of a system, which is described in the third section of this paper. Thus, we have one general definition and one general theory, but both the definition and theory may be specified to a variety of special cases, types, and kinds.

The general theory of information is built as a system of principles that represent intrinsic properties of information and information processes. The set of the main principles consists of two parts: basic ontolo gical and basic axiological principles. Ontology describes being, while axiology characterizes comprehension and evaluation of this being. Basic ontological principles of the general theory of information are formulated and investigated in the third section of the paper. They reflect the most essential properties of information as a natural, social, and technological phenomenon as well as regularities of information functioning. This provides a foundation for the mathematical development of the general theory of information.

\section{The Contemporary Situation in Information Studies and the General Theory of Information}

\subsection{The Notion of Information}

Etymologically the term information is a noun formed from the verb 'to inform,' which was borrowed in the 15th century from the Latin word 'informare,' which means 'to give form to,' 'to shape,' or 'to form.' During the Renaissance the word 'to inform' was synonymous to the word 'to instruct.' Later its meaning extended essentially and it became one of the most important technical and scientific terms. A detailed exposi tion for the etymological and historical background of the meaning of the term "information" is given by Capurro (1978). Here we are mostly interested in the scientific meaning of this term.

The outstanding American mathematician and cybernetician Norbert Wiener was the first who considered information beyond its day-to-day usage. He is one of the founders of cybernetics as a scientific discipline. The aim was to bring together similar research efforts in communication engineering, psychology, sociology, biology and medicine. From the point of view of these disciplines, it is above all the quant ity of information which, apart from message, amount of interference (noise) and coding technique, is to be accounted for. According to Wiener the transmission of information is only possible as a transmission of at ternatives, for if only a message about one possible state is to be transmitted it is most easily done by not 
transmitting any information at all. Therefore he calls for the development of a statistical theory of the amount of information, a quantity that has natural affinities to entropy in statistical mechanics. While the amount of information of a system is a measure of the degree of order, the entropy of a system is one of the measures of the degree of disorder. However, this did not solve the question of a concept of informa tion proper. Throughout his life Wiener attached special importance to finding an answer to this question. To this purpose he made use of the results of a long-term collaboration with medical scientists. His research led Wiener to make the famous statement (Wiener 1961): "Information is information, not matter or energy."

Although it is not a definition of information, this statement contained the message that the actual objects used for communication, i.e., for conveying information, are less important than information itself. Gregory Chaitin (1999) has developed this idea. He writes: "The conventional view is that matter is primary, and that information, if it exists, emerges from the matter. But what if information is primary and matter is the secondary phenomenon! After all, the same information can have many different material representations in biology, in physics, and in psychology: DNA, RNA; DVD's, videotapes; long-term memory, short-term memory, nerve impulses, hormones. The material representation is irrelevant, what counts is the information itself. The same software can run on many machines. INFORMATION is a really revolur tionary new kind of concept, and recognition of this fact is one of the milestones of this age".

Since the time of Wiener's pioneering works, information science emerged giving birth to many informa tion theories and producing a quantity of definitions of information. The birth of information theory is placed officially in 1948, when the outstanding American engineer and mathematician Claude Shannon published his first epoch-making paper. Although Shannon called what he created a theory of communication and wrote not about information itself, but about quantity of information, his research made possible to produce some definitions of information.

\subsection{Definitions of Information}

The most popular idea is that information is a message or communication. But a message is not information because the same message can contain a lot of information for one person and no information for another person. The most utilized scientific definition of information (cf., for example, (Hartley, 1928) or (Ursul, 1971) is:

(1) Information is the eliminated uncertainty.

Another version of this definition treats information as a more general essence and has the following form:

(2) Information is the eliminated uncertainty or reflected variety.

For example, Rauterberg (1995) assumes that for representation of information processes in learning systems that interact with their environment, the variety is more relevant than the concept of uncertainty reflected by probabilities in the formulas of Hartley and Shannon. But in many cases, people speak about receiving or transmitting information when the variety is undefined and there is no uncertainty. In other cases, there is variety without information.

This is illustrated by the opinions of different authors. For example, an interesting idea is suggested in the book of Knapp (1978): variety is defined in the orthogonal way to information. In non-technical language this means that variety, as a phenomenon, is essentially distinct from information. This approach correlates with what writes Wilson (1993): "In the real world ... we frequently receive communications of facts, data, news, or whatever which leave us more confused than ever. Under the formal definition these communications contain no information..." 
Both definitions (1) and (2) are based on Shannon's information theory (Shannon 1948). This theory represents a statistical approach to information and is the most popular now. However, one of the followers of Shannon, the well-known French scientist Leon Brillouin wrote that in this theory "the human aspect of in formation" is completely ignored. As a result, the statistical approach has been very misleading in social sciences and humanities. So, it was not by chance that Claude Shannon called it a theory of communica tion but not of information. Besides, Shannon himself never defined information and wrote only about the quantity of information.

\subsection{Paradoxes and Contradictions in Definitions of Information}

A vivid picture of confusion about information is given in the book of Mark Poster (1990). He begins with the statement that information "has become a privileged term in our culture that evokes a certain feature of the new cultural conjuncture and must be treated with suspicion." Describing the scientific approach, he writes that some cybernetic theorists define information in a broad sense "simply as organization of matter and energy" (Poster 1990: 28). In the narrow sense, information is that part of a communication that is not "lost" in its transmission. The part that is "lost" is noise. At the same time, Poster writes about "many forms of information: words, numbers, music, visual images" (Poster 1990: 27).

If we take such an authoritative source of definitions as The American Heritage Dictionary (1996), we see the following definitions.

Information is: 1. Knowledge derived from study, experience, or instruction. 2. Knowledge of a specific event or situation; intelligence. 3. A collection of facts or data: "statistical information." 4. The act of informing or the condition of being informed; communication of knowledge: "Safety instructions are provided for the information of our passengers." 5. (in Computer Science) A nonaccidental signal or character used as an input to a computer or communications system. 6. A numerical measure of the uncertainty of an experimental outcome. 7. (in Law) A formal accusation of a crime made by a public officer rather than by grand jury indictment.

Similar definitions of information are in the Roget's New Thesaurus: 1. That which is known about a specific subject or situation: data, fact (used in plural), intelligence, knowledge, lore. 2. That which is known; the sum of what has been perceived, discovered, or inferred: knowledge, lore, wisdom. And in both cases, there is a reference to the concept knowledge

In O'Brien (1995), which is used as a textbook at universities and colleges, it is written that the terms data and information are used interchangeably, but while data are raw material resources, information are data that has been transformed into a meaningful and useful context. In Laudon (1996) we find a similar notion of information, which is defined as an organized collection of data that can be understood.

Another definition of information is presented in Rochester (1996). According to him, information is an organized collection of facts and data. Rochester develops this definition through building a hierarchy in which data are transformed into information into knowledge into wisdom. Thus, information appears as an intermediate level leading from data to knowledge.

Ignoring that an "organized collection" is not a sufficiently exact concept, it is possible to come to a conclusion that we have an appropriate definition of information. This definition and similar ones are used in a lot of monographs and textbooks on computer science. Ignoring slight differences, we may assume that this is the most popular definition of information. This gives an impression that we actually have a working concept.

Many will say, "If such a definition exists and people who are experts in computer science use it, then what's wrong with it? Why we need something else?" To explain why this definition is actually incoherent, let us consider some examples where information is involved. The first example is dealing with a text that contains a lot of highly organized data. However, this text is written in Chinese. An individual, who does not know Chinese, cannot understand this text. Consequently, it contains no information for this person be cause such a person cannot distinct this text from a senseless collection of hieroglyphs. However, we have one and the same collection of organized data, while it contains information only for those who know 
Chinese. Thus, we come to a conclusion that information is something different from this collection of organized data.

It is possible to speculate that this collection of data is really information but it is accessible only by those who can understand the text. In our case, they are those who know Chinese. Nevertheless, this is not the case. To explain this, we consider the second example. We have another text, which is a review paper in mathematics. Three people, a high level mathematician $\mathbf{A}$, a mathematics major $\mathbf{B}$, and a layman $\mathbf{C}$, encounter this paper, which is in the field of expertise of $\mathbf{A}$. After all three of them read or tried to read the paper, they come to the following conclusion. The paper contains very little information for $\mathbf{A}$ because he already knows what is written in it. The paper contains no information for $\mathbf{C}$ because he does not understand it. The paper contains a lot of information for $\mathbf{B}$ because he can understand it and knows very little about the material that is presented in it. So, the paper contains different information for each of them. At the same time, data in the paper are not changing as well as their organization. This vividly shows that data, even with a high organization, and information have an extremely distinct nature. Structuring and re structuring cannot eliminate these distinctions.

These inconsistencies that appear when we try to apply the given definition explicitly demonstrate that this definition is not adequate. It does not reflect real situations, and we need an essentially dissimilar definition. In general, information has been considered as the following essences: as structures; processes (like becoming informed); changes in a knowledge system; some type of knowledge (for example, as personal beliefs or recorded knowledge); some type of data; an indication; intelligence; lore; wisdom; an advice; an accusation; signals; facts; acts; messages; as different things; as meaning; and as an effect like elimination of uncertainty (Brillouin 1957, Ursul 1971, Wersig/Neveling 1976, Buckland 1991, Wilson 1993, etc.).

\subsection{General Confusion}

Consequently, it is not surprising that, as it is stated in the introduction, intelligent people come to a conclusion that the main problem is that people and even experts in the field do not really know what informa tion is (Bosma 1985). Moreover, in one of his lectures the well-known American philosopher John Searle stressed that "the notion of information is extremely misleading." At the same time, the famous French mathematician Rene Thom (1975) calls the word 'information' a "semantic chameleon," that is something that changes itself easily to correspond to the environment. Various scientific and laymen imaginations about information stand often without the least explicit relationship to each other. We see that "too many" definitions may be as bad as "too little."

One more example of the general confusion about information is that some, maybe the majority of researchers, relate information only to society or, at least, to intelligent systems (cf. for example O'Brien (1995) or Laudon (1996), while others contradict information and communication, treating information as a category of solely physical systems (cf. for example Bougnoux (1995). All this demonstrates that the exist ing variety of definitions lacks a system approach to the phenomenon and for a long time there has been a need to elaborate a concept that reflects the main properties of information. This concept has to be the base for a new theory of information, giving an efficient tool for information processing and management.

However, knowing about information is not only a theoretical necessity but is a practical demand. Considering the United States of America in the information age, Giuliano (1983) states that the "informatiza tion process is very poorly understood. One of the reasons for this is that information work is very often seen as overhead; as something that is necessary but not contributory." In addition to this, there were other problems with theoretical studies of information. For example, Shannon's information theory applies only in those contexts where its precise assumptions hold, i.e., never in reality. However, experts in information studies understood that this does not imply that an attempt to create a more general theory of information should not be pursued. On the contrary it should. The existing theories are actually too restrictive.

At the same time, in spite of a multitude of papers and books concerning information and a lot of studies in this area, many important properties of information are unknown. As writes Tom Wilson (1993), " 'In- 
formation' is such a widely used word such a commonsensical word, that it may seem surprising that it has given 'information scientists' so much trouble over the years." That is one of the reasons why no adequate concept (as well as understanding) of information phenomenon has been produced by information theory till the last decade of the $20^{\text {th }}$ century.

\subsection{A New Approach}

It is possible to compare the development of information sciences with the history of geometry. At first, dif ferent geometrical objects (lines, angles, circles, triangles etc.) were investigated. When an adequate knowledge base of properties of geometrical objects was created, a new step was taken by introduc tion of the axiomatic theory that is now called the Euclidean geometry. In a similar way, knowledge obtained in various directions (statistical (Shannon 1948), semantic (Bar-Hillel/Carnap 1958), algorithmic (Solomonoff 1964; Kolmogorov 1965; Chaitin 1966), qualitative (Mazur 1984), Fisher information (Frieden 1998), economical (Marschak 1971), social (Goguen 1997, etc.) of information theory, as well as practical experience of information technology, made it possible to take a new step - to elaborate a unifying theory. It is called the general theory of information.

A general theory of information can be developed on the base of an axiomatic methodology, according to which theory is developed from a set of axioms by means of logical deduction and construction (Bur gin 2001, 2002). However, the great complexity of the phenomenon of information implies the neces sity to utilize not the standard axiomatic architecture but its more developed form. At first, the main principles are formulated. Then (on the second stage of the development of the general theory of in formation) a mathematical model and corresponding axiomatic theory are created. This mathematical theory is based on the main principles as well as on the apparatus of the theory of named sets or fundamental triads (Burgin 1990, 1991). In this mathematical context, axioms of the general information theory are introduced. These axioms are mathematical images of the main principles. They provide a possibility to construct a formal theory of information, which is exposed elsewhere.

Information studies have a two-fold aim. On the one hand, we want to understand what information is, how it exists and functions. On the other hand, to achieve this goal, it is necessary to know how to get this knowledge. In other words, we need to find how to acquire information about information properties and to derive regularities of information functioning. In accordance with this two-fold aim, the set of the main principles of the general theory of information consists of two groups: the basic ontological and basic axiological principles. Basic ontological principles reflect the most essential properties of information as a natural, social, and technological phenomenon as well as regularities of information functioning. Basic axiological principles explain how to measure and evaluate information. They systemat ize and unify different approaches, existing as well as possible, to construction and utilization of in formation measures. This, axiological aspect of the theory is as essential as the ontological one be cause methods of modern science emphasize importance of measurement and evaluation that are technical means of observation and experiment.

\section{Basic Ontological Principles of the General Theory of Information}

Ontological principles reflect major features of information existence and behaviour.

\subsection{Relativity in Information}

Traditionally, researchers tried to define information as some absolute essence that exists autonomously. Here we suggest that this may be considered only as a theoretical abstraction, while on the empirical level information is subjected to dynamical relativity. This feature of information is expressed in Principles $\mathrm{O} 1, \mathrm{O} 4$, and $\mathrm{O} 5$. 
Ontological Principle 01. It is necessary to separate information in general from information (or a portion of information) for a system $\mathbf{R}$. In other words, empirically, it is possible to speak only about informa tion (or a portion of information) for a system.

Definition 3.1. The system $\mathbf{R}$ is called the receiver of the information $\mathbf{I}$.

Why is this principle so important? The reason is that all conventional theories of information assume that information exists as something absolute, like time in the Newtonian dynamics. Consequently, this absolute information may be measured, used, and transmitted. In some abstract sense it is true, but on practice, or as scientists say, empirically, this is not so.

To demonstrate this, let us consider the following situation. We have a book in Japanese and want to ask what information it contains. For a person who does not know Japanese, it contains no information. At the same time, its information for those who know Japanese may be immense.

Another situation: let us consider a textbook, for example, in mathematics. If it is a good textbook, then it contains a lot of information for a mathematics student. However, if we show this book to a professional mathematician, she or he might say, "Oh, I know everything in this book, so it contains no informa tion for me." We will have the same result but for a different reason if we give this book to an art student who is bored with mathematics. To make the situation more evident, imagine a completely deaf and blind person who comes to a movie theatre without any devices to compensate his deficiencies. How much information this person will get there?

Being more adequate to reality than those assumptions that are made in other directions of information theory, the first ontological principle makes possible to resolve a controversy that exists in the research community of information scientists. As it is mentioned before, some of them suggest that information exists only in society, some of them relate information only to non-living systems, while others ascribe information to any phenomenon. Basing on the principle 01 , the general theory of informa tion states that if we speak about information for people, then it exists only in society because now people exist only in society. In other words, information for people does not exist without people. However, when we consider a more general situation, then we see that information exists in everything and it is only a problem how to extract it. For example, for a biologist any living creature contains information. Stars contain information for astronomers, while stones contain information for geologists. This understanding is reflected by Principle $\mathrm{O} 2$.

It is necessary to remark that the relativity of information has been observed and discussed by some re searchers. For example, defining social information, Goguen (1997) writes that his definition ties an item of information to a particular group through a particular relationship of accountability for a particur lar interpretation. However, the same configuration of signs could very well be interpreted by different groups in different ways, giving rise to different items of information. In this approach, it takes work to interpret signs as information, and this work is necessarily done in some particular context, making use the resources available and within the constraints imposed in that context.

Similar ideas, but in much broader context are suggested by Jumarie (1986), who hypothesizes that in formation is related to a basic triplet (triad): the system $S$ that is the material medium where informa tion is physically defined; the universe of discourse $U$ where information is semasiologically defined; and the observer $\mathrm{R}$ who considers $\mathrm{S}$ and $\mathrm{U}$ in his own subjective framework.

\subsection{The Essence of Information}

Thus, the first principle explicates an important property if information, but says nothing about what information is. This is done by the second principle that exists in two forms.

Ontological Principle 02. In a broad sense, information I for a system $\mathbf{R}$ is any essence causing changes in the system $\mathbf{R}$.

This principle has several consequences. First, it demonstrates that information is closely connected with transformation. Namely, it means that information and transformation are functionally similar because 
they both imply changes in a system. At the same time, they are different because information is a cause of a change while transformation is the change itself.

Second, it explains why information influences society and individuals. Namely, reception of information implies transformation. In this sense, information is similar to energy. Moreover, according to Principle $\mathrm{O} 2$, energy is a kind of information in a broad sense. This exactly corresponds to the Carl Friedrich von Weizsäcker's idea (cf. for example Flükiger 1995) that energy might in the end turn out to be information. In its turn, the von Weizsäcker's conjecture explains the exact correspondence between such characteristic of energy as the thermodynamic entropy, which is given by the Boltzmann-Planck formula $S=k \cdot \ln P$, and such characteristic of information as the quantity of information, which is given by a similar Hartley-Shannon formula I = K.In N.

Third, Principle $\mathrm{O} 2$ makes it possible to separate different kinds of information. For example, any person as well as any computer has many kinds of memory. It is even supposed that each part of the brain has several types of memory agencies that work in somewhat different ways, to suit particular purposes (Minsky 1986). It is possible to consider each of these memory agencies as a separate system and to study differences between information that changes each type of memory. This would help to understand the interplay between stability and flexibility of mind, in general, and memory, in particular.

It is true, to be sure, that an adequate theory, whether of the information or anything else, must be in significant accord with our common ways of thinking and talking about what the theory is about. Else there is the danger that theory is not about what it purports to be about. But, on the other hand, it is wrong to expect that any adequate and reasonably comprehensive theory will be congruent in every respect with common ways of thinking and speaking about its subject, just because those ways are not themselves usually consistent or even entirely clear. To achieve this goal, we introduce information in the strict sense, which is based on the concept of an infological subsystem of a system.

Definition 3.2. A subsystem IF(R) of the system $\mathbf{R}$ is called an infological system of $\mathbf{R}$ if $\operatorname{IF}(\mathbf{R})$ contains infological elements.

Infological elements are different kinds of structures (Burgin 1991, 1997). Let us take as a standard example of infological elements knowledge, data, images, ideas, fancies, abstractions, beliefs, etc. If we consider only knowledge and data, then the infological system is the system of knowledge. This sys tem is very important, especially, for intelligent systems. The majority of researchers believe that information is intrinsically connected to knowledge (cf. Flückiger 1995). Consequently, we take the sys tem of knowledge of $\mathbf{R}$ as a model example of an infological system IF(R) of an intelligent system $\mathbf{R}$. It is called in cybernetics the thesaurus $T h(\mathbf{R})$ of the system $\mathbf{R}$. When $\mathbf{R}$ is a material system, its infological subsystem $\mathrm{IF}(\mathbf{R})$ consists of three components: a material component, which is a system of physical objects; a functional structure realized by the material component; and the system of infological elements. For example, the material component of the infological subsystem of a human being is her/his brains. The corresponding functional structure is her/his mind. Infological elements in this case will be units of knowledge of the individual. Another example of an infological system is the memory of a computer. Such a memory is a place in which data and programs are stored.

Remark 3.1. An arbitrary complex system $\mathbf{R}$ has, as a rule, different infological subsystems. Fixing one of these subsystems, we determine what is information for $\mathbf{R}$ and changing our choice of $\mathrm{IF}(\mathbf{R})$ we change the scope of information entities.

For example, computers have different kinds of memory: processor registers, addressed memory, main storage, buffer storage, external storage, working storage etc. Each of them or any combination of them may be considered as an infological subsystem of a computer $\mathbf{R}$. If the processor registers are treated as an infological subsystem, then no program (even such that is kept in the main storage of this computer) has information for $\mathbf{R}$ until execution of instructions of this program begins.

Ontological Principle 02a. Information in the strict sense or, simply, information for a system $\mathbf{R}$, is everything that changes the infological system $\operatorname{IF}(\mathbf{R})$ of the system $\mathbf{R}$.

This principle and Remark 1 imply that for a complex system there are different kinds of information. Each infological system determines a specific kind of information. For example, information that causes 
changes in the system of knowledge is called cognitive information. This kind of information is crucial for pedagogy.

Different approaches to information as a phenomenon and to information theory support this principle. Here we discuss only two examples of such approaches, which have not been considered before. One is given by pragmatic information introduced by Ernst Ulrich von Weizsäcker (1974) and further developed by Von Lucadou (1987, 1994), Kornwachs (1996), Weinberger (2001) and some others. This concept relies on the wo notions firstness ("Erstmaligkeit") or originality or novelty, and confirma tion ("Bestätigung") or redundancy to already known. Weizsäcker suggests that a message that does nothing but confirm the prior knowledge of a receiver will not change its structure or behaviour. Thus, with confirmation up to $100 \%$, a message gives no pragmatic information. On the other hand, a message providing only original/novel material completely unrelated to any prior knowledge also will not change structure or behaviour of the receiver, because the receiver will not understand this message. Thus, with firstness up to $100 \%$, a message gives no pragmatic information. Only a relevant mixture of firstness and confirmation allows the receiver to get pragmatic information from the message.

Thus, pragmatic information, according to Weizsäcker, is related to changes of structure or behaviour of the receiver. This corresponds to Principle $\mathrm{O} 2$, and thus, the theory of pragmatic information is a spe cial case of the general theory of information. Taking thesaurus, i.e., the system of knowledge, as an infological system, adding some principles and developing them further, we obtain the theory of pragmatic information.

The psychologist von Lucadou $(1987,1994)$ applied pragmatic information to problems of psychology. He writes: "The model of pragmatic information (MPI), which is a candidate for a non-classical model of psychology, predicts that the behavior of a non-classical system depends on the conditions of its observation. The exchanged pragmatic information (meaningful information) ties the "observer" (e.g., a person) and the "observed" (e.g., a machine) together and creates an "organizational closed system." It is assumed that this process produces non-local correlations between the observer and the observed."

In such a way, pragmatic information acts on the observed system. This entirely corresponds to Principle 02a. However, Principle 02a allows to go further and to consider two opportunities of change. In one case, behavior of the system under observation changes. It corresponds to an action on the behaviofal infological system. In the other case, changes only information that we get from the system under observation. It corresponds to an action on the representational infological system.

The second example is social information in the sense of Goguen (1997). He suggests the following definition:

An item of information is an interpretation of a configuration of signs for which members of some social group are accountable.

According to the general theory of information, a configuration of signs is a carrier of information, while in terpretation as an action is the change caused in some social group. At the same time, interpretation as some text (symbolic configuration) is an element of the cognitive infological system of this group.

Remark 3.2. In what follows, we use the terminus "information" instead of "a portion of information" when it does not cause misunderstanding.

\subsection{Information and Energy}

Ontological Principle O2a implies that information is not of the same kind as knowledge and data, which are structures in the sense of Burgin $(1997,2002)$. To explain this, we need to clarify the distinction between matter and energy. As it is written in Lindsay (1971), the concept of energy is one of the greatest ideas ever created by the mind of man. Energy is traditionally interpreted as ability to do work (cf., for example, Asimov 1959 or Beiser 1962).

There is a traditional misinterpretation that the famous Einstein's formula $E=m c^{2}$ implies that matter is equivalent to energy. Actually, this formula only states that the quantity of energy in some portion of 
matter is proportional to such property/characteristic of matter as mass related to the same portion of matter. The coefficient of proportionality is $\mathrm{c}^{2}$. By the way, the formula $E=\mathrm{mc}^{2}$ is similar to the formula of kinetic energy $E=1 / 2 \mathrm{mV}^{2}$. However, nobody has suggested that the latter formula implies equivat ence of matter and energy. Thus, the formula $E=m^{2}$ also does not imply that matter and energy are the same. Theoretical physics allows two possibilities: energy is a property of material bodies or en ergy and matter are different essences. We suggest that energy and matter are different but intrinsically connected with one another essences: matter cannot exist without energy (at least, zero energy), while energy is always contained in physical bodies. Taking matter as the name for all physical substances as opposed to energy and the vacuum, we have the relation that is represented by the following diagram.

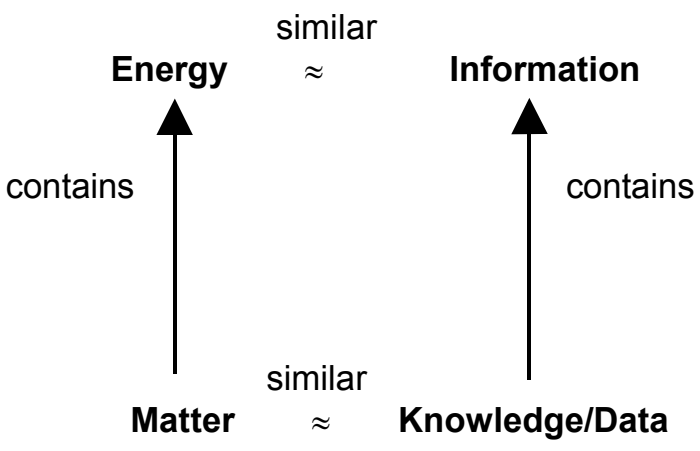

Figure 1: Information/Energy Diagram

Similarity of matter and knowledge (reflected in figure 1) means that they may be considered in a static form, while energy and information exist only in (actual or potential) dynamics. In addition, similarity of energy and information signify that both these entities cause change in systems: energy does this in physical systems, while information does this in structural systems such as knowledge and data (Burgin 1997). In other words, the diagram states that information is related to knowledge and data as energy is related to matter. More exactly, this relations holds for cognitive information that changes such infological system as thesaurus or system of knowledge. However, there are other types of information, for instance, emotional information (Burgin 2001), which influence emotional infological systems of people.

\subsection{Knowledge and cognitive information}

The distinction between knowledge and cognitive information implies that transaction of information (for example, in a teaching process) does not give knowledge itself. It only causes such changes that may result in the growth of knowledge. This correlates with the approaches of Dretske (1981) and MacKay (1969), who declare that information increases knowledge and knowledge is considered as a completed act of information.

However, the general theory of information differs from this conception of information because it demonstrates that information transaction may result also in the decrease of knowledge (Burgin 1994). It happens so, for instance, when we get false information.

According to Flückiger (1995), in modern information theory a distinction is made between structural-at tributive and functional-cybernetic types of theories. Representatives of the former approach conceive in- 
formation as structure, like knowledge or data. Adherents of the latter understand information as functionality, functional meaning or as a property of organized systems.

Our conception of information belongs to the functional approach because it is possible to consider many kinds of conventional information as a property of knowledge. At the same time, functional conceptions look incoherent with respect to the structural approach, and we have the question whether it is worth to have two notions of information as they have historically emerged or it is necessary to preserve only one of them to achieve higher integrity of sciences.

To find a grounded answer, we have both historical and methodological arguments. From history, we know that when physics was only emerging as a science, there was a lot of controversy on the problem what was energy. However, the necessity to build exact mathematical models of physical phenomena res ulted in making the term 'energy' sufficiently precise and giving other names to similar phenomena. Consequently, the term 'information' also has to be sufficiently precise. It might be possible only if we choose either a functional or a structural approach to the definition of information.

In addition to this, we have the following methodological arguments explaining why it is not reasonable, logical, and even consistent to consider information as a kind of knowledge or knowledge as a kind of in formation.

- Only some kinds of information are related to knowledge. There are other kinds, which, for example, are connected to emotions, which are often contrasted to knowledge (Burgin 2001, 2002).

- According to the methodological principle that is called Ockham's razor, it is not necessary to call one and the same thing by two names knowledge and information. One name is sufficient, and knowledge is better in this case. Besides, as it is demonstrated in the previous section, properties of information are essentially different from properties of knowledge.

- In addition to this, it is not logical to consider information as some structure because structural aspects of the world are covered by other scientific concepts. Consequently, like matter is only a vessel for energy, but not energy itself, static structures (knowledge, data, signs, texts, etc.) are only carriers for in formation, while information reflects dynamic aspects of reality.

So paraphrasing Wiener, we may say that information is not knowledge and knowledge is not informa tion.

Remark 3.3. The symbol IF may be considered as an operator that is defined on a space of systems of some chosen kind (may be on the space of all systems). Being applied to a system $\mathbf{R}$, the operator IF defines in $\mathbf{R}$ its infological subsystem IF(R). However, as a rule, this is a multivalued or variable operator because it is possible that $\mathbf{R}$ has several infological subsystems.

Remark 3.4. It is possible to argue that the concept of an infological system is too ambiguous and fuzzy. However, ambiguity may be a positive property if you can use it. For example, if you can control and change ambiguity, it becomes not an ambiguity but a parameter that is utilized to tune and control the sys tem under consideration. This is just the case with the infological system in the general theory of informa tion. Thus, it is natural that a human being has not the same infological system as a biological cell or a computer.

Remark 3.5. As it is mentioned above, in modern information theory there is the structural-attributive direction, representatives of which conceive information as structure, like knowledge or data, variety, order, and so on (cf., for example, MacKay 1969, Nauta 1970, Seifert 1968, Dretske 1981, Flückiger 1995). According to the general theory of information such structures are not information per se. They are infological elements, which are structural carriers of information. Consequently, all theories that belong to the structural-attributive direction are included in the scope of the general theory of information through the subtheory of infological systems and elements.

The fact that information influences human behaviour to such a great extent is a consequence of the fact that human infological systems (or subsystems) control human actions. The latter is a necessary trait for adaptation of an individual both in nature and society. 


\subsection{Types of Information}

A possibility to choose an infological system in a different way is very beneficial. It helps to discover existence of different types and kinds of information. Each type of information corresponds to some type of infological system. In what follows, we consider only information in the strict sense.

Definition 3.3. If information, or more strictly, a portion of information I changes only $\operatorname{IF}(\mathbf{R})$ (and nothing more), then I is called ( a portion of) pure information.

Definition 3.4. If information I changes $\operatorname{IF}(\mathbf{R})$ and some other parts of $\mathbf{R}$ then $I$ is called extended information or more strictly, (a portion of) extended information.

For example, when a boxer receives a knock, it is extended information because it adds to the boxer's knowledge that he has received a knock and at the same time it changes his physical state. Sometimes it is difficult to make an exact distinction between pure and extended information. For example, some individual watched TV and what she saw impressed her so much she changed her life. In particular, being fat she stopped eating too much and became slender and active. Thus, information that this person had gotten watching TV had changed her even physically. Consequently, on one hand this information is pure be cause it had changed only knowledge of this person. On the other hand, it looks, according to Definition 3.4, like an extended information.

Even a text may contain extended information. Let us consider a letter $L$ that contains information about death of some person $\mathbf{A}$. Imagine such a situation. A close relative $\mathbf{R}$ of $\mathbf{A}$ receives this letter, reads it and then has a stroke because the news from the letter was so horrible for him. Thus, we can state that the let ter $L$ contained for $\mathbf{R}$ some portion of extended information.

Definition 3.5. If information I (in the general sense) does not change $I F(R)$, then I is called quasiinformation for $\mathbf{R}$.

To facilitate understanding of these terms, we give the corresponding Venn diagram:

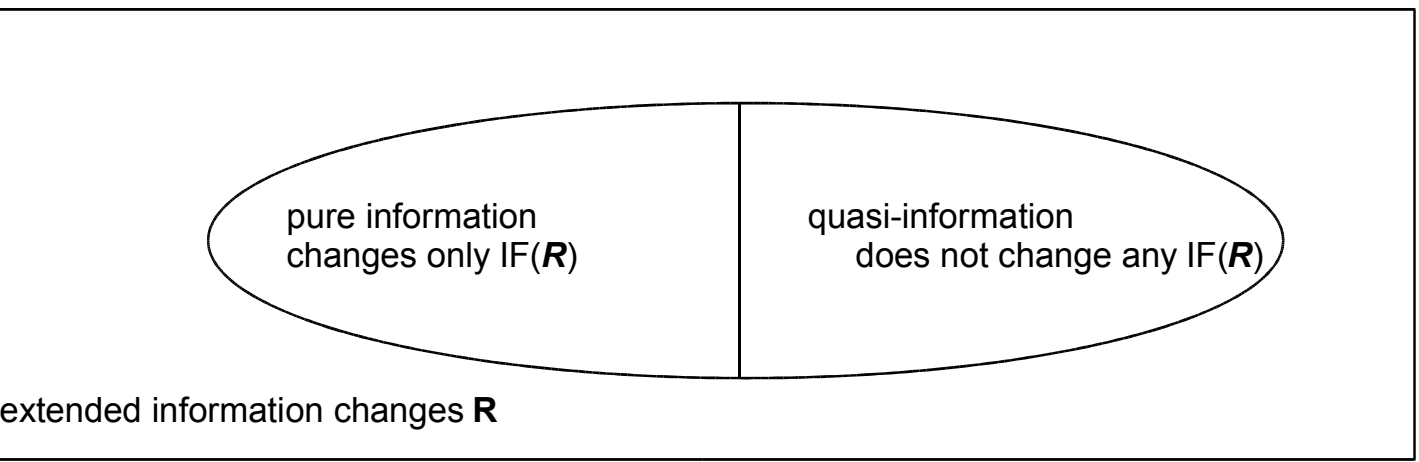

Figure 2: Venn diagram

In what follows, we do not consider quasiinformation, i.e., such generalized information, which does not include pure information. However, this concept is very important for the entertainment industry, which suf fers from a lot of reproaches for disseminating quasiinformation.

\subsection{Information Dynamics}

Let I be some portion of information for a system $\mathbf{R}$.

Ontological Principle 03. There is always some carrier $\mathbf{C}$ of the information $\mathbf{I}$.

Really, people get information from books, magazines, TV and radio sets, computers, and from other people. To store information people use their brains, paper, tapes, and computer disks.

Carriers of information belong to three classes: material, mental, and structural. For example, let us consider a book. It is a physical carrier of information. However, it contains information only because some 
meaningful text is printed in it. Without this text it would not be a book. The text is the structural carrier of information in the book. Besides, the text is understood if it represents some knowledge. This knowledge is the mental carrier of information in the book.

Two of the three types of information carriers are related to elements of the basic triplet (triad) of Jumarie (1986). The system $S$ that is the material medium where information is physically defined corres ponds to the physical carrier of information. The universe of discourse $U$ where information is semasiologically defined corresponds to the mental carrier of information. For adherents of the materialistic approach Principle $\mathrm{O} 3$ must be changed to a stronger version:

Ontological Principle OM3. There is some substance/substratum C that contains information I.

Definition 3.6. This substance $\mathbf{C}$ is called the physical carrier of $\mathbf{I}$.

The first three ontological principles ((O1)-(O3) or (O1)-(OM3)) imply that information connects the carrier $\mathbf{C}$ with the system $\mathbf{R}$ and is a component of the following triple structure

(1) $(\mathbf{C}, \mathbf{I}, \mathbf{R})$

As a rule, there is some channel through which information comes from $\mathbf{C}$ to $\mathbf{R}$. For example, The carrier $\mathbf{C}$ of $\mathbf{I}$ is a piece of paper and $\mathbf{R}$ is a person reading the text written on $\mathbf{C}$. Then the corresponding channel is the space between the paper and the eyes of the person.

The structure (1) is a special case of the following more general structure that is called a named set or a fundamental triad (Burgin, 1990; 1991; 1997):

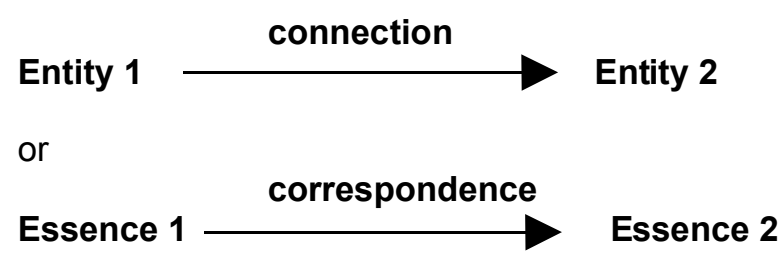

Figure 3: Fundamental triad

In the fundamental triad, Entity 1 (Essence 1) is called the support, the Entity 2 (Essence 2) is called the reflector (or the set of names) and the connection (correspondence) is called the reflection (or the naming relation) of the fundamental triad (named set).

There are many fundamental triads in which Entity 1 is some set, Entity 2 consists of the names of the elements from the Entity 1 and elements are connected with their names by the naming relation. This explains the name "named set" that was for an essential time applied to fundamental triads (Burgin 1990, 1991). A standard model of this structure is a set of people who constitute the carrier, their names that form the set of names, and the naming relation consists of the correspondences between people and their names.

People meet fundamental triads constantly in their everyday life. An example is given by the traditional scheme of communication. Communication is extensively studied as process of information transmission and reception. According to Shannon, in order for messages to be transmitted, we need a communication system.

Shannon describes the components of the communication system as follows:

1. The information source (or message source) produces messages or parts of messages intended for a particular destination.

2. On the basis of the message, the transmitter produces a sequence of signals such that they can be transmitted over a channel.

3. The channel is merely the medium used to transmit the signal from transmitter to receiver. During transmission the signals may be perturbed and distorted by a so-called noise source.

4. The receiver usually performs the reverse operation to the transmitter, reconstructing if possible the original message from the signals. 
5. The destination is the addressee of the message and can be either a person or a thing. It requires a priori knowledge about the information source, which enables it to understand the message transmitted. In any case, the destination must know the set of signs available to the information source.

Communication process as a total event has been a subject of many studies. Many models or structural descriptions of the communication event have been suggested to aid understanding of the general organization of the total event. This makes possible to classify and to describe the parts of the process and to indicate how they fit together and interact in the process. Models provide clues that permit predictions of be haviour and thus stimulate further research. There are static and dynamic models. Static models represent the system in which the communication goes on. Dynamic models feature functioning of such a system.

The simplest static model consists of three elements: sender, receiver, and channel (Figure 4a). The simplest dynamic model consists of three elements: sender, receiver, and message (Figure 4b).

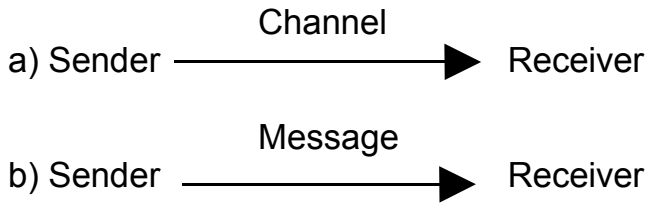

Figure 4: The simplest models of a communication event.

a) The static model; b) The dynamic model.

A more detailed, synthetic model incorporates both static and dynamic features of the process. It is presented in the Figure 5. Connection here consists of three components: the physical communication media (in particular, a channel), the message as the pragmatic part of communication, and structural communication means. The latter include language(s), which is (are) used for coding and decoding the message; emission, transmission, and reception algorithms (software systems) etc. Physical systems that perform emission, transmission, and reception are parts of the physical communication media.

\section{Connection \\ Sender $\longrightarrow$ Receiver}

Figure 5: The synthetic model of a communication event.

It is necessary to remark that principles of a theory do not allow one to achieve the necessary exact ness. That is why, mathematical structures are utilized and axioms of the general theory of information are introduced. These axioms give an exact mathematical reflection of the main principles and provide for elaboration of a general axiomatic theory of information, which is based on the theory of named sets (fundamental triads). Fundamental triads are used for construction of the mathematical part of the general theory of information. But before doing this, we need all main principles of this theory.

Ontological Principle 04. A transaction of information goes on only in some interaction of $\mathbf{C}$ with $\mathbf{R}$.

This interaction may be direct or indirect, i.e. it is realized by means of some other objects. The property of information explicated in Principle $\mathrm{O} 4$ may look evident. However, it has important consequences, For example, if you know that some information has passed from system to another and want to find how it happened, you have to look for a channel of transaction. Although, if it is known only that the second system possesses the same information as the first one, it not necessary that it has been a transmission. It might be possible that the same information has been created by the second system. Existence of a channel makes transmission possible but does not necessitates it.

The next principle is a clarification of Principle O4.

Ontological Principle 04a. A system $\mathbf{R}$ receives information I only if some carrier $\mathbf{C}$ of I transmits I to the system $\mathbf{R}$ or $\mathbf{R}$ extracts this information from $\mathbf{C}$ through some channel ch. 
Here, we have two ways of information transaction: transmission and extraction. Transmission of information is the passive transaction with respect to $\mathbf{R}$ when $\mathbf{R}$ receives information and active transaction with respect to $\mathbf{C}$ when $\mathbf{C}$ transmits information. Extraction of information is the active transaction with respect to $\mathbf{R}$ when $\mathbf{R}$ extracts information and passive transaction with respect to $\mathbf{C}$ when information is taken from $\mathbf{C}$. When the carrier $\mathbf{C}$ is the system $\mathbf{R}$ itself, then we have the third type of information operations - information processing. It includes information transformation and production (Burgin 1997b).

The two ways of information exchange reflect interesting regularities of education. There is an essential difference between Western and Eastern approaches to education. The main principle of the Western tra dition is that a teacher comes to students to teach them. Contrary to this, the main principle of the Eastern tradition is that a student comes to teacher to learn from him. This means that the Western approach is based on information transmission, while the Eastern approach stems from information extraction.

Two more principles explicate dynamic properties of information processes.

Ontological Principle 05. A system R accepts information I only if the transaction causes corresponding transformations.

For example, if after reading this paper, your knowledge remains the same, you do not accept cognitive information from this text. That is why the concern of the people from the entertainment industry how their production influences the intended audience is the greatest importance to the industry. The general theory of information can explain many features of this impact.

Ontological Principle O6. One and the same carrier $\mathbf{C}$ can contain different portions of information for one and the same system $\mathbf{R}$.

Really, let us take some person $\mathbf{A}$ as the system $\mathbf{R}$ and a book written in Japanese as the system $\mathbf{C}$. At first, $\mathbf{A}$ does not know Japanese and $\mathbf{C}$ contains almost no information for $\mathbf{A}$. After some time, $\mathbf{A}$ learns Japanese, reads the book $\mathbf{C}$ and finds in it a lot of valuable information for himself. Note that knowing Japanese $\mathbf{A}$ is, in some sense, another person.

In other words, if you want to convey some information to an audience efficiently, you have to prepare this audience to acceptation of the transferred information. This is essentially important for a teacher (Bur gin 2001). His exposition must be not only logically, but also psychologically ordered and organized.

There are many examples supporting Principle O6 when unprepared community (an intended receptor) did not accept even the highest achievements of human intellect and creativity (transmitted information). Thus, it is known that contemporaries did not accept many outstanding works of art as well as great dis coveries. Only consequent generations understood the greatness of what had been done before. As examples, we can take the great composer Mozart, who died in poverty, the great mathematicians Galois and Abel, who wrote outstanding works but were neglected and died at a very young age as a result of such an attitude.

One more example of misunderstanding gives the life of the great English physicist Paul Dirac. He was well known and respected by physical community when he theoretically discovered a positive "electron", which was later called positron. However, other physicists did not understand Dirac's achievement and even mocked at him in spite of general recognition that he is an outstanding physicist.

At the same time, the great German mathematician Gauss made one of the most outstanding discoveries of the $19^{\text {th }}$ century, the discovery of the non-Euclidean geometry. Nevertheless, he did not want to publish his discovery because correctly considered the contemporary mathematical community unprepared to the comprehension of this discovery.

The last three principles reflect only such situations when transformation of an infological system takes place. However, it is important to know and predict properties of these transformations, for example, to evaluate the extent or measure of transformations.

\section{Conclusion}

Thus, the main ontological principles of the general theory of information show that: 
- although information is relative, it is not necessarily related to meaning or knowledge (in more detail see Burgin (2001)

- information transmission is not a simple action but consists of three inseparable constituents: informa tion emission, transaction, and reception (in more detail see (Burgin 1997a);

- information is not constructed by the receiver: what may be constructed is knowledge, meaning, beliefs etc.

- information is an objective essence, which depends on a receiving system.

To explain this paradoxical feature of information, we can compare the situation with the concept of time in physics. According to relativity theory (Einstein et al. 1923), time is an objective characteristic of physical systems. However, time, or more exactly, relations between times in different systems, depends on the ret ative velocity of one system with respect to another system.

To conclude it is necessary to remark that originality of the general theory of information is demonstrated its discoveries of such types of information that were not even mentioned before by existing information theories. For instance, it allows us to discover new types of information and to demonstrate that there are three main types of information in biological systems: cognitive, emotive or conditioning, and regulative in formation (Burgin 2001, 2002). Cognitive information is intrinsically related to knowledge and thus, it is what people understand when they use the name "information." Two other types are new and help to understand many phenomena in system functioning. Their properties have different applications in education and entertainment industry.

\section{References}

Agre, P. (1995) Institutional Circuitry: Thinking about the Forms and Uses of Information. In: Information Technologies and Libraries, December, pp. 225-230.

Asimov, I. (1959) Words of Science, and the History behind Them. Boston. Houghton Mifflin Co.

Bar-Hillel, Y. and Carnap, R. (1958) Semantic Information. In: British J. of Philosophical Sciences, Vol. 4, No. 3, pp. 147-157.

Bar-Hillel, Y. (1964) Language and Information, Selected Essays on their Theory and Application. Reading, Massachusetts. Addison-Wesley.

Barwise, J./Perry, J. (1983) Situations and Attitudes. Cambridge, Massachusetts/London, England. MIT Press.

Bateson, G. and Ruesch, J. (1951) Communication: The Social Matrix of Psychiatry. New York. W.W.Norton and Co.

Beiser, A. (1962) The Mainstream of Physics. Reading/Palo Alto/London .Addison Wesley.

Bell, D. (1980) The Social Framework of the Information Society. In: Dertouzos, M./Moses, J. (Eds) (1980) The Computer Age: A Twenty-Year View. Cambridge. MIT Press, pp. 163-211.

Bosma, H. (1983) Information Quality rather then Information Quantity, In: Information Policy and Scientific Research". Amsterdam. Elsevier, pp. 99-106 .

Bougnoux, D. (1995) La Communication contre I'Information. Paris. Hachette.

Bower, G.H./Hilgard, E. R. (1975) Theories of Learning. Englewood Cliffs, NJ. Prentice-Hall.

Bowker, G. (1994) Information Mythology. In: Bud-Frierman, Lisa (Ed.) (1994) Information Acumen: The Understanding and Use of Knowledge in Modern Business. London. Routledge.

Brillouin, L. (1956) Science and Information Theory. New York. Academic Press Inc. Publishers.

Buckland, M. (1991) Information and Information Systems. New York. Praeger .

Burgin, M.S. (1990) Theory of Named Sets as a Foundational Basis for Mathematics. In: Structures in Mathematical Theorie". San Sebastian, pp. 417-420

Burgin, M.S. (1991) What the Surrounding World is Built of. In: Philosophical and Sociological Thought, No. 8 (in Russian and Ukrainian).

Burgin, M.S. (1994) Evaluation of Scientific Activity in the Dynamic Theory of Information. In: Science and Science of Science, No. 1, pp. 124-131.

Burgin, M.S. (1997) Fundamental Structures of Knowledge and Information, Academy for Information Sciences, Kiev (in Russian).

Burgin, M.S. (1997b) Information Algebras. In: Control Systems and Machines, No.6, pp. 5-16 (in Russian).

Burgin, M. (2001) Information in the Context of Education. In: Journal of Interdisciplinary Studies, Vol. 14, pp. 155-166.

Burgin, M. (2002) Information, Organization, and System Functioning, In: Proceedings of the $6^{\text {th }}$ World Multiconference on Systemics, Cybernetics and Informatics. v. 2, Orlando, Florida, pp. 155-160 
Capurro, R. (1978) Information. München. Saur.

Capurro, R./Fleissner, P./Hofkirchner, W. (1999) Is a Unified Theory of Information Feasible? In: Hofkirchner, Wolfgang (Ed.) (1999) The Quest for a unified theory of information, Proceedings of the $2^{\text {nd }}$ International Conference on the Foundations of Information Science. Amsterdam. Gordon \& Breach. pp. 9-30.

Chaitin, G.J. (1966) On the Length of Programs for Computing Finite Binary Sequences. In: J. Association for Computing Machinery, Vol. 13, No. 4, pp. 547-569.

Chaitin, G.J. (1977) Algorithmic information theory. In: IBM Journal of Research and Development, Vol. 21, No. 4, pp. 350-359.

Chaitin, G.J. (1999) The Unknowable. Berlin/Heidelberg/New York. Springer.

Dretske, F. I. (1981) Knowledge and the Flow of Information. Oxford. Basil Blackwell.

Einstein, A./Lorentz, H.A./Weyl, H./Minkowski, H. (1923) Principles of Relativity. Dover.

Fisher, R. A. (1950) Contributions to Mathematical Statistics, New York, Wiley

Fleissner, P./Hofkirchner, W. (1996) Emergent Information: Towards a unified theory of information,. In: Biosystems, Vol. 38, No. 2/3, pp. 243-248.

Flükiger, D.F. (1995) Contributions towards a Unified Concept of Information. Doctoral Thesis. University of Bern

Frieden, R.B. (1998) Physics from Fisher Information. Cambridge. Cambridge University Press.

Fuchs, C./Hofkirchner, W./Klauninger, B. (2001) The Dialectic of Bottom-Up and Top-Down-Emergence in Social Systems. In: Proceedings of the Conference "Problems of Individual Emergence". Amsterdam. Online: http://www.selforganization.org/results/papers/pdf/hsicpaper8.pdf

Giuliano, V.E. (1983) The United States of America in the Information Age. In: Information Policy and Scientific Research. Amsterdam. Elsevier, pp. 59-76

Goguen, J.A. (1997) Towards a Social, Ethical Theory of Information. In: Social Science Research, Technical Systems and Cooperative Work, Erlbaum, pp. 27-56.

Harkevich, A. (1960) On the value of information. In: Problems of Cybernetics, Vol. 4, pp.2-10.

Hartley, R.V.L. (1928) Transmission of Information. In: Bell System Techn. Journal 7, No. 3, pp. 535-563

Hofkirchner, W. (1999)Towards a Unified Theory of Information, In: 15e Congrès International de Cybernétique Namur. Namur. pp. 175-180.

Hofkirchner, W. (2001) The Hidden Ontology: Real World Evolutionary Systems Concept as Key to Information Science. In: Emergence, Vol. 3, No. 3, pp. 22-41.

Jumarie, G.M. (1986) Subjectivity, Information, Systems. New York/London/Paris. Gordon and Breach.

Kolmogorov, A.N. (1965) Three approaches to the definition of the quantity of information. In: Problems of Information Transmission, No. 1 , pp.3-11.

Kornwachs, K. (1996) Pragmatic Information and the Generation of Knowledge. In: Interdisciplinary Approaches to a New Understanding of Cognition and Consciousness. Ulm. Ulmer Universitätsverlag, pp. 33-74.

Kornwachs, K. (2001) Instantaneous Availability of Cultural Goods - a Driving Force for Superficiality and Loss of Knowledge? In: Institut für Technikfolgenabschätzung und Systemanalyse (ITAS) Proceedings of the Int. Conference: Innovations for an E-Society - Challenges for Technology Assessment. Berlin.

Laudon, K.C. (1996) Information Technology and Society. Belmont, California. Wadsworth P.C.,

Lindsay, R.B. (1971) Basic Concepts of Physics. New York/Toronto/London. van Nostrand Reinhold Co.

Von Lucadou, W. (1987) The Model of Pragmatic Information. In: Proceedings of the 30th Parapsychological Association Convention, pp. 236-254.

Von Lucadou, W. (1994) The Endo- Exo-Perspective - Heaven and Hell of Parapsychology. In: Proceedings of the 37th Parapsychological Association Convention, pp. 242-252.

Luhmann, N. (1995) Social Systems. Stanford, CA. Stanford University Press.

Lyon, D. (1988) The Information Society: Issues and Illusions. Cambridge. Polity.

MacKay, D. M. (1969) Information, Mechanism and Meaning. Cambridge, Massachusetts. MIT Press.

Marschak, J. (1973) Value and cost of information systems. Production theory. In: Proc. Internat. Sem., Univ. Karlsruhe, Karlsruhe, 1973. Berlin. Springer, pp. 335-358.

Marschak, J. (1971) Economics of information systems. In: J. Amer. Statist. Assoc., Vol. 66, 1971, pp. $192-219$.

Mazur, M. (1970) Jakosciowa Teoria Informacji, Warszawa, PAN (in Polish).

Minsky, M. (1986) The Society of Mind. New York. Simon and Schuster.

Nauta, D. (1970) The Meaning of Information. Paris. Mouton/The Hague.

O’Brien, J.A. (1995) The Nature of Computers. Philadelphia/San Diego. The Dryden Press.

Poster, M. (1990) The Mode of Information. Chicago. University of Chicago Press,

Rauterberg, M. (1995) About a Framework for Information and Information Processing of Learning systems. In: Information System Concepts: Towards a Consolidation of Views, Proc. IFIP Intern. Working Conference on information Systems Concepts. London/Glasgow/ New York. Chapman \& Hall.

Rochester, J.B. (1996) Using Computers and Information. Indianapolis. Education and Training. 
Seiffert, H. (1968) Information über die Information, Verständigung im Alltag, Nachrichtentechnik, wissenschaftliches Verstehen, In formationssoziologie, das Wissen des Gelehrten. München. Beck

Shannon, C.E. (1948) The Mathematical Theory of Communication. In: Bell System Technical Journal, Vol. 27, No. 1, pp. 379-423; No. 3, pp.623-656.

Solomonoff, R. (1964) A Formal Theory of Inductive Inference. In: Information and Control, Vol. 7, No. 1, pp. 1-22.

Stone, A.R. (1997) The War of Desire and Technology at the Close of the Mechanical Age. Cambridge, Massachussetts. MIT Press. Tohm, R. (1975) Structural Stability and Morphogenesis. New York. Benjamin.

Ursul, A.D. (1971) Information. Moscow. Nauka (in Russian).

Wheeler, J.A. (1990) Information, Physics, Quantum: The Search for Links. In: Complexity, Entropy, and the Physics of Information. Redwood City, CA. Addison-Wesley.

Weinberger, E.D. (2001) A Theory of Pragmatic Information and Its Application to the Quasispecies Model of Biological Evolution. In: nlin.AO/0105030 (electronic edition: http://arXiv.org)

von Weizsäcker, E.U. (1974) Erstmaligkeit und Bestätigung als Komponenten der pragmatischen Information, im Offene Systeme I, Beiträge zur Zeitstruktur von Information, Entropie und Evolution. Stuttgart. Klett, pp. 82-113.

Wiener, N. (1961) Cybernetics, or Control and Communication in the Animal and the Machine. New York/London. MIT Press/Wiley. Wilson, T. (1993), Trends and Issues in Information Science - a General Survey. In: Media, Knowledge and Power. London/Sydney. Groom Helm, pp.407-422. 